\title{
THE RADIATION FOCUSING BY THE ARRAY OF WAVEGUIDE BASED ELEMENTS WITH CONTROLLABLE REFLECTION
}

\author{
Aleksandr Eremeev ${ }^{1}$, Vladimir Antipov \\ National Research Tomsk State University, the Department of Radiophysics, 634050, Tomsk, Russia
}

\begin{abstract}
The authors propose a solution of the problem of radiovision using the reflective array, each element of which can change the reflection coefficient under the action of external control voltage. The focusing abilities of flat reflection array of monochromatic radiation were studied to solve the problem of radiovision. The array element based on waveguide with a controlled reflection coefficient was developed. The phase shift switching is $180^{\circ}$.
\end{abstract}

\section{Introduction}

Modern radiovision systems are applied for numerous purposes: to control the quality of materials, buildings and constructions as well as for medical applications. The development of computing technologies provided the application of the radiotomography techniques for the remote non-destructive testing and the diagnostics of the internal structure of radiosemitransparent media and the shape recovery of radio opaque objects. The task of radiotomography is the transformation of the data obtained from the multiangle scanning of target objects into the 3D visualization [1]. The electronically and electromechanically scanned arrays are developed to obtain the multiangle projections of the wave field. However, the design and development of such arrays are complicated and expensive task, as it requires the development and control of numerous microwave transceiver channels.

The development of the electronically scanned arrays widely prevails nowadays over the electromecanically scanned ones. Together with conventional phased arrays, the arrays of elements with controllable phase of reflection are extensively applied. The reflection elements are usually based on ferrite phase shifters, micromechanical systems or varactor diodes [2].

The phase switching of the array reflection coefficients enables the simulation of the Fresnel zone plate that provides the focusing of feeder radiation at a certain point. The waves from two neighboring Fresnel zones are counter-phased. The electronic scanning can be performed in a certain angle sector with the change in geometrical arrangement of zone rings [3-4]. In this case, the only transceiver at fixed position can be used.

${ }^{1}$ Corresponding author: aleksandr_eremeev_93@mail.ru 


\section{Formulation of the problem}

In this research, the authors propose a solution of the problem of radiovision using the reflective array, each element of which can change the reflection coefficient by the external voltage control. The detailed task scheme presented in Figure 1. A transmitted spherical wave be propagated in a free space defined in the Cartesian coordinate system. The transmitter (1) is placed at $Z$ axis and at the $h$ height from the reflective array (2). The test object (3) is placed above the array.

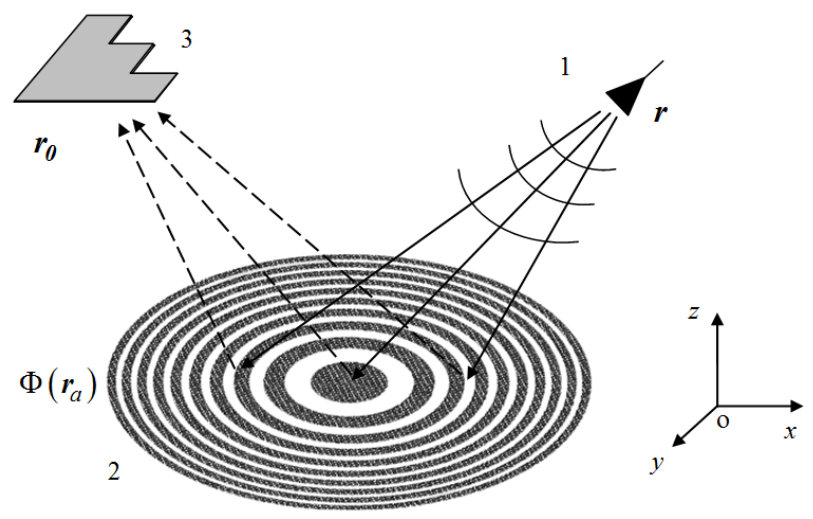

Figure 1. Task scheme.

Set the focusing point coordinates as $\left(x_{0}, y_{0}, z_{0}\right)$. Then, the Fresnel zone boundaries are defined by the equation:

$$
\sqrt{z_{0}^{2}+\left(x-x_{0}\right)^{2}+\left(y-y_{0}\right)^{2}}+\sqrt{h^{2}+x^{2}+y^{2}}=n \frac{\lambda}{2}
$$

where $n$ is the number of the Fresnel zone boundary, $\lambda$ is the wavelength of the sounding signal.

Previously, two problems were solved [5]. The equation for reflection coefficient distribution was obtained, which provided the radiation focusing at the certain point. Also, the equation describing the field at the receiver point was obtained as well. The using of the $2 \mathrm{D}$ reflective array with controlled reflection coefficient to focus the monochromatic radiation for radiotomographic application were analysed. The numerical simulations with a test object approved the recovery of the test object shape.

\section{Waveguide element of the reflection array}

A waveguide was used as an element of the reflection array. The scheme of the waveguide array element and its pilot prototype are presented in Figure 2. 

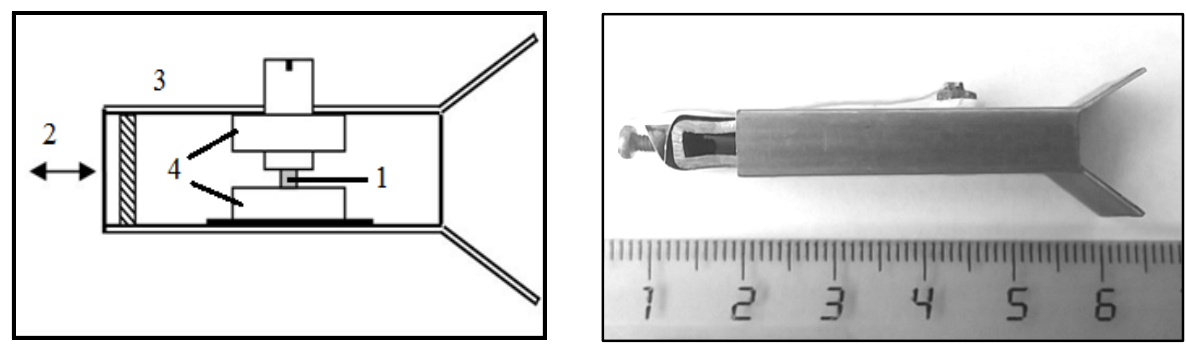

Figure 2. Array element structure (1- semiconductor diode capacitor, 2- shorting plunger, 3- waveguide, 4 -impedance transformer) and its external view.

The varactor diode was installed into the waveguide section $11 \times 5.5 \mathrm{~mm}$ in size. The waveguide impedance could be switched from low to high by altering the varactor capacity through the voltage adjustment. In that case, the installed varactor diode could either shunt the waveguide or transmit the propagated wave to the shortening plunger. In the latter, the phase shift to be set as $180^{\circ}$ by the shorting plunger position.

To match the waveguide, its narrow walls were cut to make the horn-shaped aperture with bent wide waveguide walls. That technique reduces the reflection as well as increases the effective area of the array. The impedance transformer was built of two symmetrically inserted conductive discs, one of which was insulated from a waveguide wall with a dielectric pad. The varactor diode was soldered to the end of the screw which was extended through the non-insulated disc to the contact with the insulated one. The control voltage was applied to the insulated disc. The waveguide element is simply constructed that makes possible its volume production as well as provides its cost competitiveness with printed phase shifters.

\section{The numerical and experimental results}

The array element experimentally demonstrated the reproducibility of characteristics. The graph illustrating the $180^{\circ}$ phase shift of the reflection coefficient is presented in Figure 3.

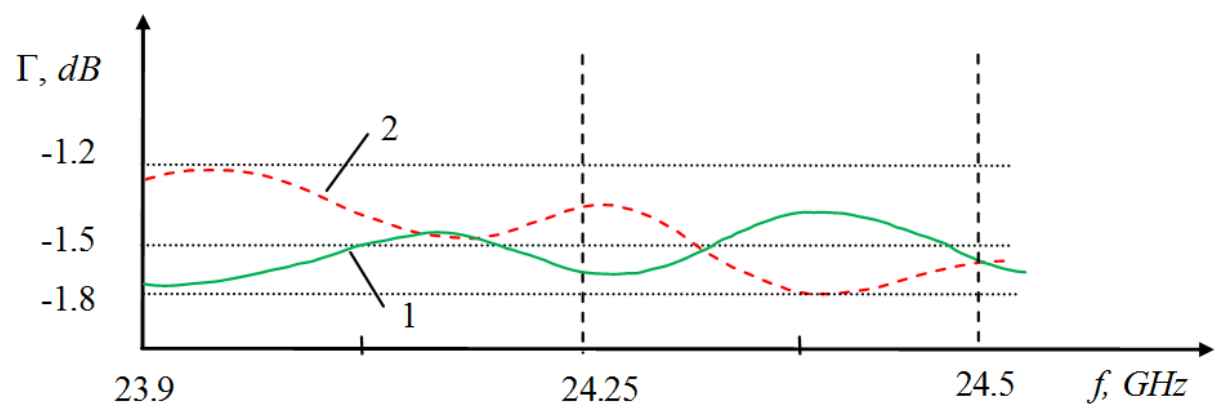

Figure 3. Reflection coefficient in the waveguide element: 1 - control voltage applied to the varactor diode, 2 - no voltage applied.

The full line graph (1) corresponds to the signal reflected when the shift voltage was applied to the varactor diode. The dashed line (2) illustrates the signal without the voltage shift. The array structure required the special holders, with 10 waveguide elements in each holder. The holders are set one above the other (Figure 4, $a$ ). 


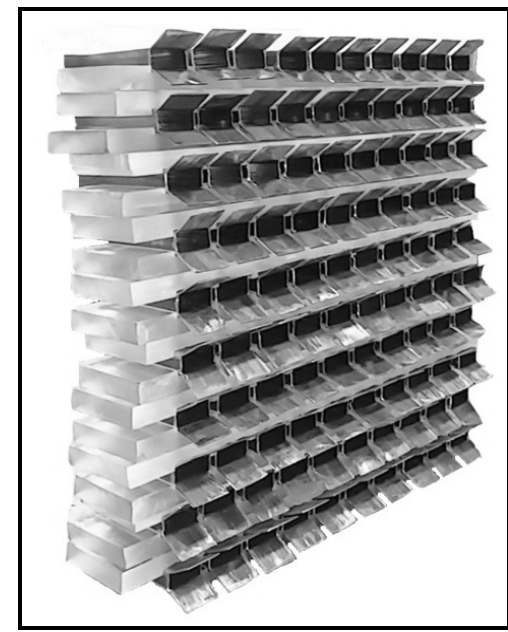

a

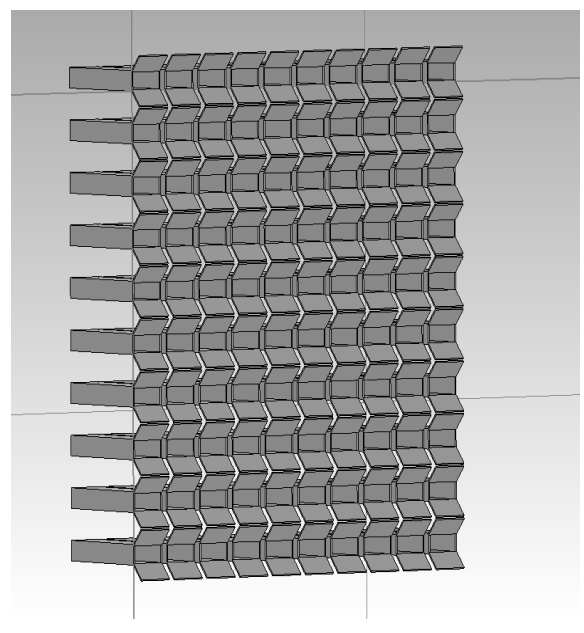

b

Figure 4. Reflection array sample in the size of 10x10 elements, and its numerical model.

To develop the experimental sample of the reflection array, the waveguide element and the array were simulated in CST Microwave Studio. Array model is presented in Figure 4,b.

The distribution of the reflection coefficient $\Phi\left(\boldsymbol{r}_{\mathrm{a}}\right)$ was predefined, which was calculated by the algorithms [5] corresponding to the array size. The calculations were performed for the frequency of $24 \mathrm{GHz}$. The distribution of the reflection coefficient, which provides the focusing at the coordinates $(0,0,10)$ is presented in Figure 5.

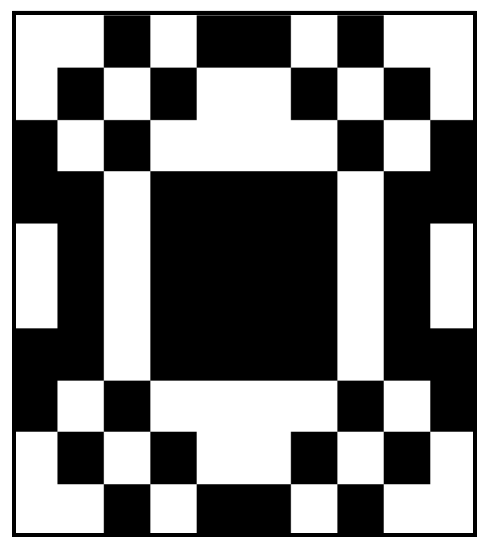

Figure 5. The Fresnel zone distribution.

The field focused with the waveguide-based reflection array is presented in Figure 6. It was calculated in CST Microwave Studio. 

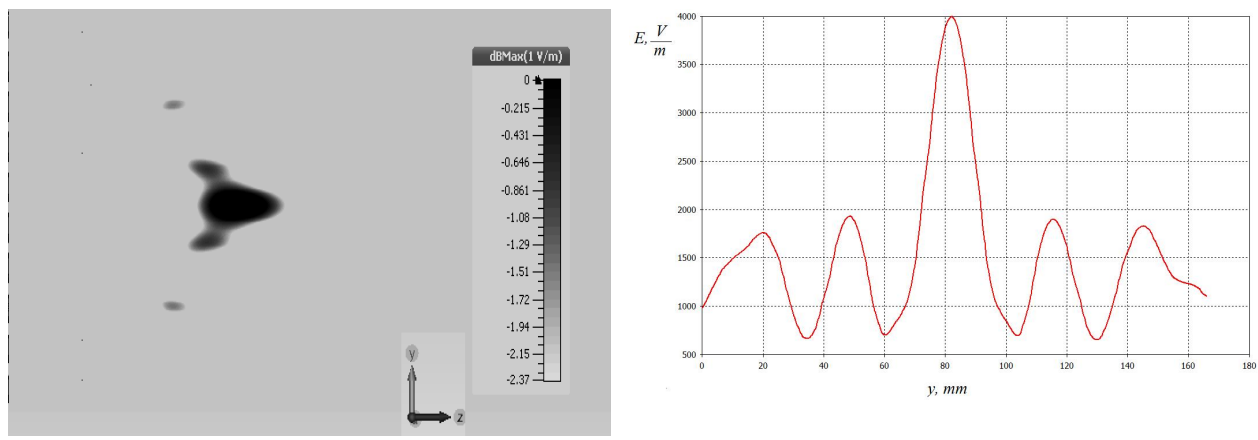

a)
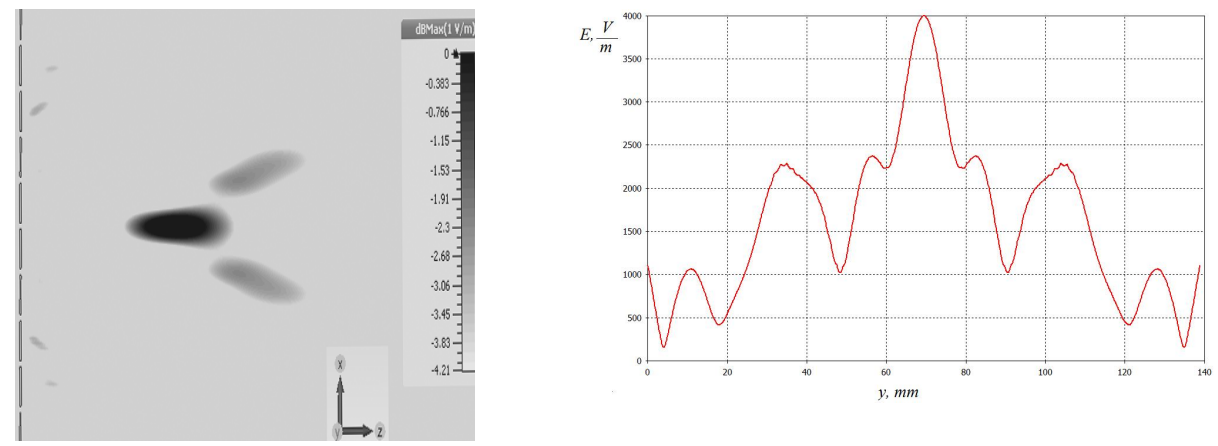

b)
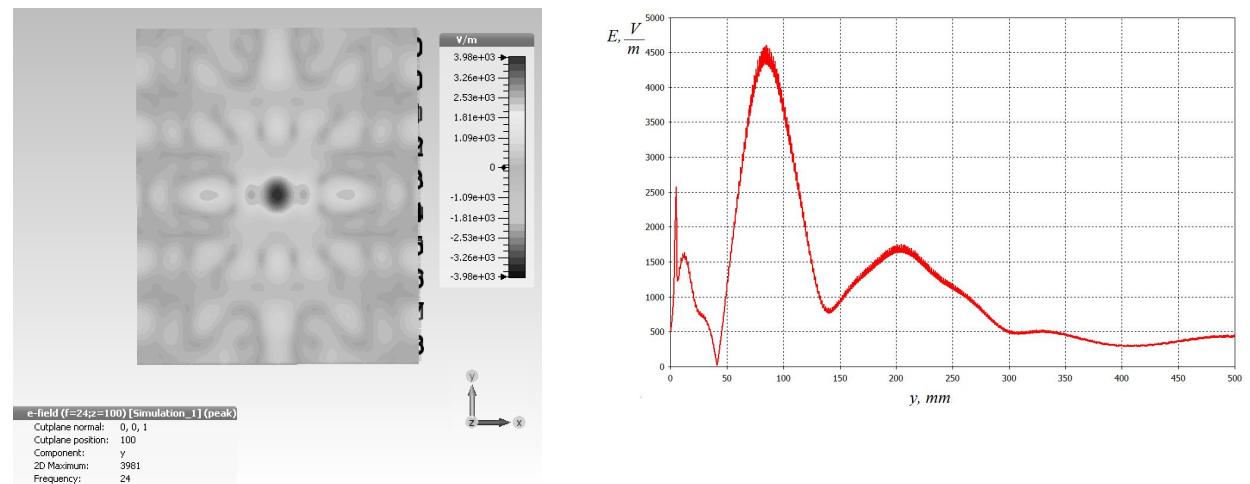

c)

Figure 6. Radiation focusing with the reflection array.

The field distribution in the $y z$ plane is presented in Figure $6, a$. Figure $6, b$ presents the field distribution in the $x z$ plane. Figure $6, c$ shows the field distribution in the $x y$ plane as well as the field strength variation depending on the distance from the array. It is indicated that the field was focused at a certain point $(0,0,10)$ which the distribution of the reflection coefficient corresponded to (Figure 5). 


\section{Conclusion}

So, the capability of the flat reflection array to focus a monochromatic radiation at a certain point were studied. The element of the array based on waveguide with a controlled reflection coefficient was developed. The controlled phase shift is $180^{\circ}$. The array model based on waveguide elements was simulated in CST Microwave Studio. These simulations proved the monochromatic radiation focusing with the array made up of waveguide elements. A hardware and software complex to control the array is being currently developed. The proposed development of the reflection tomographic setup is considered to be a Russian analogue of the equipment from other countries. The proposed structure of the reflection array provides the high-rate focusing at a certain point.

\section{Acknowledgment}

The research was supported by the Russian Ministry of Education and Science as a part of the state order No 3.694.2014/K.

\section{References}

[1] Y. Kan, Y. Zhu, Q. Fu, MATEC Web of Conferences 56, 02001 (2016) doi: 10.1051/matecconf/20165602001

[2] S. V. Hum, J. Perruisseau-Carrier, IEEE Transactions on Antennas and Propagation 62, 183 (2014) doi: 10.1109/TAP.2013.2287296

[3] G. Leon, L. Herran, M. Munoz, F. Las-Heras, Y. Hao, Prog. Electromagn. Res. 54, 125 (2014)

[4] M. Hajian, G. Vree, L. Ligthart, IEEE Antennas Propag. Mag. 45, 13 (2013) doi: 10.1109/MAP.2003.1252806

[5] S. Shipilov, A. Eremeev, V. Yakubov, J. Phys.: Conf. Ser. 671, 012052 (2016) doi: $10.1088 / 1742-6596 / 671 / 1 / 012052$ 